Cómo citar este artículo en Chicago: Castrillón Gallego, Catalina y Andrés Villegas. "Posibilidades y límites de la extensión cultural universitaria, 1935-1954: revistas y emisoras universitarias en Colombia”. Escritos 30, no. 64 (2022): 89-102. doi: http://doi.org/10.18566/escr.v30n64.a06

Fecha de recepción: 29.07 .2021

Fecha de aceptación: 13.12.2021

\title{
Posibilidades y límites de la extensión cultural universitaria, 1935-1954: revistas y emisoras universitarias en Colombia
}

\author{
Possibilities and limits of university cultural extension, 1935-1954: \\ university journals and radio stations in Colombia
}

\author{
Catalina Castrillón Gallego ${ }^{1}$ \\ Andrés Villegas ${ }^{2}$
}

\begin{abstract}
RESUMEN
Este artículo indaga por el uso de medios de comunicación para el desarrollo de actividades de extensión cultural que dieron la Universidad de Antioquia y la Universidad Pontificia Bolivariana entre 1935 y 1953. A través de la crítica de fuentes documentales se describe y analiza como ambas instituciones, siguiendo referentes de los ámbitos nacional e internacional, desarrollaron en estos años estrategias como la publicación de revistas y la creación de emisoras que se convirtieron en importantes herramientas para extender y amplificar las labores desarrolladas dentro de sus respectivos claustros académicos. La revisión de la Revista Universidad de Antioquia (1935), la Revista Universidad Pontificia Bolivariana (1937) y los boletines de programas y las parrillas de programación de Emisora Cultural Universidad de Antioquia (1933) y Radio Bolivariana (1948), permitió plantear que ambas universidades a través de sus emisoras, revistas y aulas intentaron entregarle a la ciudad, la región y el país su versión de lo que entendía por cultura. Al hacerlo se enfrentaron a sus propias limitaciones, principalmente, a la debilidad de su infraestructura y la escasez de sus recursos para participar en un mercado cada vez más competitivo, profesional, diverso y que se dirigía hacia una dirección decididamente comercial; a esto se sumaban, las tensiones y contradicciones inherentes a proyectos de este tipo, que se enfrentaban al reto de difundir expresiones culturales que, paradójicamente, eran consideradas como minoritarias y, en ese sentido, difícilmente aceptables y disfrutables más allá de las capas con una educación formal prolongada.
\end{abstract}

Palabras clave: Cultura; Medios de comunicación; Extensión cultural; Revistas culturales; Radio universitaria.

1 Profesora Asociada de la Universidad Pontificia Bolivariana, Colombia. Doctora en Historia y miembro del grupo de investigación Epimeleia de la misma universidad. Correo electrónico: catalina.castrillon@upb.edu.co.

2 Profesor Asociado de la Universidad Nacional de Colombia - Sede Medellín. Doctor en Historia y miembro del grupo de investigación "Historia, espacio y cultura” de la misma universidad. Correo electrónico: aavilleg@unal.edu.co. 


\begin{abstract}
This article investigates the use of communication media for the development of cultural extension activities carried out by the Universidad de Antioquia and the Universidad Pontificia Bolivariana between 1935 and 1953. On the basis of critical reviews on diverse documentary sources, it is described and analyzed how both institutions, following national and international benchmarks, developed strategies such as the publication of journals and the creation of radio broadcast stations over the years, which became relevant tools to extend and amplify the work carried out within their respective academic cloisters. The review of the Revista Universidad de Antioquia (1935), the Revista Universidad Pontificia Bolivariana (1937) and the program bulletins and programming grids of Emisora Cultural Universidad de Antioquia (1933) and Radio Bolivariana (1948), allowed us to state that, through their stations, magazines and classrooms, both universities tried to give the city, the region and the country their version of what they understood by culture. In doing so, they faced their own limitations, mainly the weakness of their infrastructure and the scarcity of their resources to participate in an increasingly competitive, professional, diverse market that was heading in a decidedly commercial direction. Added to this were the tensions and contradictions inherent to projects of this type, which faced the challenge of disseminating cultural expressions that, paradoxically, were considered minority and, in that sense, hardly acceptable and enjoyable beyond the layers with a prolonged formal education.
\end{abstract}

Keywords: Culture; Media; Cultural Extension; Cultural Journals; University Radio Station.

\title{
Introducción
}

〔C Necesitamos cultura y más cultura!", este era el llamado de José J. Sierra, director de la emisora de la Universidad de Antioquia, en la revista de la misma institución en 1937, puesto que "nada mejor puede ofrecer por ahora la Universidad a sus hijos más dilectos, obreros, artesanos y empleados, que una estación de carácter netamente cultural". ${ }^{3}$ Dos aspectos llaman de inmediato la atención del lector contemporáneo: por un lado, los actores sociales que son declarados favoritos de la institución, y por otro, el medio a través del cual se demuestra esta predilección.

La posible extrañeza del lector se desvanece si consideramos que la década de 1930 fue un momento de grandes transformaciones políticas, económicas, sociales y culturales en el país. ${ }^{4}$ Estas hacían parte de un proceso que podría ser sintetizado bajo la idea de la crisis de la ciudad letrada ${ }^{5}$ y la emergencia de la ciudad masificada. ${ }^{6}$ No se trataba de un proceso simple ni unidireccional, tampoco implicaba el declive o la desaparición de la "alta cultura". Lo que estaba en juego era cómo la confluencia de la urbanización, modernización y masificación redefinía las prácticas y representaciones de y sobre la cultura, y cómo modificaba las relaciones entre las instituciones y los productores culturales canónicos y las personas que estaban por fuera de los circuitos culturales de mayor legitimidad. En este sentido, la presencia cada vez más notoria de los obreros, artesanos y empleados redefine lo que se comprende por cultura, que deja de ser un atributo de las élites para constituirse en un vínculo por construir entre sectores sociales. ${ }^{7}$

3 José J. Sierra, "Radiodifusión cultural", Revista Universidad de Antioquia, no. 13 (1937): 33.

4 Malcolm Deas, "Las claves del periodo", en Colombia: Mirando hacia dentro. Vol. 4 1930-1960, coord. por Eduardo Posada Carbó y Malcolm Deas (Bogotá: Taurus, 2015), 17.

5 Ángel Rama, La ciudad letrada (Santiago de Chile: Tajamar, 2004), 162.

6 José Luis Romero, Latinoamérica, las ciudades y las ideas (Medellín: Universidad de Antioquia, 1999), 385.

7 Graciela Montaldo, Museo del consumo: Archivos de la cultura de masas en Argentina (Buenos Aires: Fondo de Cultura Económica, 2016), 20. 
En este contexto, surge la preocupación por la extensión cultural en Colombia. La necesidad de difundir la cultura fue objeto de un consenso casi incuestionable, pero las formas concretas de hacerlo propiciaron álgidas discusiones. Buena parte de los desacuerdos sobre cómo hacerlo estaban relacionados con la misma ambigüedad de lo que se pretendía extender: la cultura. Renán Silva ha planteado al respecto:

La cultura aparece como una totalidad en la cual es posible distinguir dos elementos. Un conjunto de producciones pertenecientes a la más elevada esfera del quehacer humano, tal como se expresa en las formas elaboradas del "espíritu", y una especie de suelo nutricio, de verdades esenciales, reencarnación de lo más auténtico que tiene un pueblo, una suerte de ocultas raíces ancestrales, una forma de invariante, y por lo tanto una materia muy poco histórica, que opera como la base de construcción de cualquier manifestación cultural que no quiera extraviarse y romper con un destino histórico fijado de antemano en el pasado. ${ }^{8}$

La extensión cultural pretendía hacer descender ese conjunto de la elevada esfera en la que residía el suelo nutricio que representaba el pueblo. Desde esta perspectiva, era posible definir la extensión cultural como "aquellas actividades que tienden, mediante ciertas influencias exteriores, a hacer del mayor número de colombianos seres humanos efectivamente cultos". ${ }^{9}$ Las producciones en cuestión eran diversas e incluían conferencias, conciertos, exposiciones artísticas, exhibiciones cinematográficas, emisión de programas radiales y publicación de libros, revistas y periódicos.

La Oficina de Extensión Cultural del Ministerio de Educación Nacional cumplió un papel protagónico en este campo, tanto bajo los gobiernos liberales (1930-1946) como bajo los gobiernos los conservadores (1946-1953). ${ }^{10}$ Las universidades fueron también lugares centrales a la hora de extender la cultura. Como instituciones de educación superior, pertenecían por derecho propio a "las elevadas esferas del espíritu" al ser vitales en la producción cultural y en la reproducción de sus productores. En la década de 1930, esta función social empezó a pensarse como central y necesaria, pero insuficiente en tanto no irrigaba ese "suelo nutricio" que corría el riesgo de tornarse estéril ante la ausencia de cuidados. Así, las universidades asumieron el compromiso de proyectarse por fuera de sus claustros, como clamaba José J. Sierra, y para ello recurrieron a los medios masivos, tanto externos, en que buscaron y encontraron un espacio, como propios que empezarían a crear en esta década. Al respecto, en un artículo significativamente titulado "La universidad y la cultura", se planteaba como tarea fundamental de todo gobierno la difusión de los "beneficios de la cultura a todas las masas", para lo cual se apelaba a las universidades, que, en el caso de la Universidad de Antioquia, contaba para esta misión con la biblioteca general, la imprenta, la radiodifusora, los museos de ciencias naturales, botánica y etnología, revistas, boletines y ciclos de conferencias. ${ }^{11}$

Este artículo se concentra en las revistas y emisoras de la Universidad de Antioquia y de la Universidad Católica Bolivariana (Universidad Pontificia Bolivariana a partir de 1945) entre 1935 y 1954. El primer hito corresponde a la creación de la Revista Universidad de Antioquia, y el segundo al año en el cual Alfonso Mora Naranjo, codirector desde su fundación, es relevado de su cargo, a lo que se suma el inicio

8 Renán Silva, República Liberal, intelectuales y cultura popular (Medellín: La Carreta, 2004), 25.

9 República de Colombia, Memoria de Educación Nacional 1939 (Imprenta del Estado Mayor General, 1939 ), 73.

10 Renán Silva, “La cultura”, en Colombia: Mirando hacia dentro. Vol. 4 1930-1960, coord. por Eduardo Posada Carbó y Malcolm Deas (Bogotá: Taurus, 2015), 277.

11 “La universidad y la cultura”, Revista Universidad de Antioquia, no. 103 (1951): 589. 
de las transmisiones de televisión que cambiarán, con el transcurrir de los años, el ecosistema medial y sus relaciones con la extensión cultural. Nos interesa en particular mostrar las posibilidades que abrió la idea de extender la cultura, pero también sus límites. Estos estuvieron dados por la escasez de medios y recursos, las elevadas tasas de analfabetismo que dificultaron el acceso a los impresos, la creciente dinámica comercial de los medios de comunicación que favoreció el crecimiento de las empresas editoriales y las emisoras privadas sobre las públicas, los vaivenes de la acción del Estado central y las contradicciones de las universidades en sus esfuerzos por alcanzar un público más amplio que el de sus estudiantes, profesores y egresados.

\section{Las revistas universitarias}

A pesar de su extensa trayectoria, poco frecuente para una publicación seriada en Colombia, nuestro conocimiento de la Revista Universidad de Antioquia está prácticamente limitado a un artículo publicado hace más de tres décadas. ${ }^{12}$ Como ya se mencionó, Alfonso Mora Naranjo, quien era el director de la biblioteca universitaria, fue su primer codirector, al lado de Clodomiro Ramírez, rector de la universidad. La figura del primero será central en tanto dirigirá la publicación durante 115 números. El número inaugural correspondió a los meses de marzo-mayo de 1935, aunque paradójicamente se presentó como una publicación mensual, lo que pocas veces se cumplió.

En su artículo programático "Nuestros propósitos”, Ramírez señalaba que era una continuación de los Anales de la Universidad de Antioquia y trazaba como objetivos fomentar el espíritu de investigación científica y promover el intercambio de publicaciones educativas y culturales, en especial entre los países indoamericanos. Agregaba: "Las conferencias culturales, el radio y el libro, son las tres palancas que vamos a mover para tratar de levantar cada día más el nivel cultural de nuestra juventud". ${ }^{13}$ Una juventud que, a su juicio, estaba atónita ante el derrumbamiento de los sistemas que los seres humanos de generaciones anteriores habían levantado.

Esta misma preocupación por la difusión cultural y la juventud marcó el inicio de la Revista Universidad Católica Bolivariana en 1937. En esta, también los rectores ejercieron como directores de la publicación; monseñor Manuel José Sierra dirigió los primeros 18 números y monseñor Félix Henao Botero 100 números (entre el 19 y el 118). En el artículo anónimo "Definición" con el que se abrió el primer número de la revista, se señalaba: "Iniciamos la publicación de la revista, tierra de siembras intelectuales, instrumento de combate, poder de renovación, órgano de la Universidad Católica Bolivariana”, ${ }^{14}$ a lo que se sumaba que se difundirían principios y doctrinas ajustadas a las enseñanzas católicas, en tanto no se reconocía ninguna oposición entre ciencia y fe.

La comparación entre estos artículos permite observar algunas semejanzas y diferencias. En ambas, hay un interés por extender las actividades y los resultados científicos que como universidades desarrollaban,

12 Santiago Londoño Vélez, “La Revista Universidad de Antioquia”, Boletín Cultural y Bibliográfico 26, no. 18 (1989): 42-49.

13 Clodomiro Ramírez, "Nuestros propósitos", Revista Universidad de Antioquia, no. 1 (1935): 5.

14 “Definición”, Revista Universidad Católica Bolivariana 1, no. 1 (1937): 3. 
también una preocupación por la juventud en un momento de incertidumbre nacional, pero, sobre todo, mundial, dada la proximidad de una nueva guerra que cuestionaba a la civilización occidental en su conjunto. Además, se hace evidente que la segunda revista consideraba necesaria la defensa de la doctrina católica y el planteamiento de la complementariedad de la ciencia y la religión, que tendrá, en ocasiones, una formulación radical: la verdadera ciencia se basa en los principios cristianos, sobre todo, aquella que pertenece a las disciplinas que hoy llamaríamos ciencias humanas. Por su parte, la Revista Universidad de Antioquia planteaba una posición secular en la cual el fomento del espíritu científico y el elevamiento de la cultura de la juventud podía buscarse sin mencionar lo que en ese tiempo recibió el nombre de la cuestión religiosa.

Es necesario recordar que la Universidad Católica Bolivariana surgió de la polémica que se presentó con la Reforma Constitucional de 1936, en especial, por su artículo 14 que buscaba garantizar la libertad de enseñanza, el cual fue interpretado por un grupo de profesores y estudiantes de la Facultad de Derecho de la Universidad de Antioquia, simpatizantes de lo que Arias Trujillo denomina catolicismo integral, ${ }^{15}$ como la puerta de entrada a la difusión de importantes errores en las cátedras universitarias, a lo cual se sumaron las denuncias de persecución a profesores y estudiantes cercanos al Partido Conservador y la Iglesia católica por autoridades gubernamentales.

No obstante, esta polémica no implicó una pugna directa entre las revistas. En Universidad de Antioquia, es posible encontrar de manera recurrente artículos de la pluma de miembros del clero secular y regular como el hermano Daniel y el sacerdote Roberto Jaramillo Arango, quienes también publicaban en la revista católica. Monseñor Henao Botero participó con sus escritos en la Revista Universidad de Antioquia y fue objeto de un homenaje fúnebre. ${ }^{16}$ Intelectuales reconocidos por su catolicismo como el chileno Clarence Finlayson y los colombianos René Uribe Ferrer, Emilio Robledo, Belisario Betancur, José María Bravo, Cayetano Betancur y Abel Naranjo Villegas, entre otros, colaboraron con ambas revistas. No obstante, quienes participaron directamente en la fundación de la Universidad Católica Bolivariana como profesores (Bravo y Betancur) o como estudiantes (Naranjo Villegas) cesaron sus colaboraciones en la Revista Universidad de Antioquia durante varios años; por ejemplo, Cayetano Betancur publicó en los dos primeros números (1935) y en el sexto (1936), y no lo volvió a hacer hasta 1941.

Los libros y las revistas reseñados en ambas publicaciones no difieren excesivamente y la estructura y tipo de sus secciones es similar. Si bien la Revista Universidad de Antioquia no incluye artículos apologéticos al catolicismo, tampoco publicó artículos que se prestaran para polémicas en ese campo. Al tiempo, la Revista de la Universidad Católica Bolivariana dio espacio tanto para la crítica como para la publicación de escritores polémicos, por su vida como Porfirio Barba Jacob, por su muerte como José Asunción Silva o por su carácter relativamente vanguardista como León de Greiff. Al respecto, con motivo de la entrega del Premio Vergara y Vergara se exaltó el reconocimiento otorgado a Tomás Carrasquilla, "toda vez que ningún escritor nacional presenta como él los caracteres de autonomía estética e interpretación directa de nuestro pueblo"; ${ }^{17}$ pero, al discutir el segundo premio, se agregaba:

15 Ricardo Arias Trujillo, El episcopado colombiano: Intransigencia y laicidad, 1850-2000 (Bogotá: Universidad de los Andes, 2003), 148

16 "En homenaje a monseñor Sierra”, Revista Universidad de Antioquia, no. 44 (1941).

17 “El Premio Vergara y Vergara de literatura nacional”, Revista Universidad Católica Bolivariana 1, no. 3 (1937): 376. 
Nadie ignora que se hicieron manipulaciones de diverso orden para crear y fortalecer una atmósfera hostil y una opinión animadversa contra las obras presentadas por la generación literaria de los últimos y los penúltimos. El caso de León de Greiff aclara nuestras afirmaciones. El tomo de versos "Variaciones alrededor de nada" alcanzó en un principio los mejores juicios del jurado y las más propincuas posibilidades de éxito. Pero los cenáculos veteristas y algunos valores intelectuales de fin de siglo organizaron la oscura conspiración contra el autor de los "Relatos". ${ }^{18}$

Tal vez la diferencia más ostensible entre ambas publicaciones está condensada en los artículos políticos, en los que, en general, se debate y critica el comunismo, y que aparecen con alguna frecuencia en la publicación católica y no en la revista laica que evita la actualidad política colombiana. Una diferencia más de grado que de fondo será que esta última revista se concentró más decididamente en la extensión de las ciencias y la literatura, mientras la primera difundió artículos científicos, incluyendo en esta categoría a los del campo de las humanidades, pero no publicó literatura en el cuerpo de la revista, sino que le dio un lugar en una sección anexa: el "Cuadernillo de poesía”, al cual se sumará luego una sección de arte.

La Revista Universidad de Antioquia en su primer número publicó el artículo programático referido, varios ensayos sobre problemas históricos, jurídicos, filosóficos, políticos y literarios, poemas, el sumario de las revistas recibidas por la biblioteca de la universidad (de la cual la revista dependía) y una sección de "Vida universitaria". El segundo número separó los artículos de carácter ensayístico de las "investigaciones", continuó con la sección de "Vida universitaria" e introdujo una sección para publicar textos de autores canónicos de las letras nacionales como José Eusebio Caro, Rafael Pombo y Miguel Antonio Caro.

Durante los diecinueve años revisados de esta publicación, sus secciones cambiaron de nombre y la revista parecía estar buscando una estructura que no acababa de encontrar; sin embargo, hay una constante que es posible sintetizar de la siguiente manera: artículos de fondo, tanto de carácter ensayístico como un poco más especializados sobre una gran diversidad de temas que están, en general, vinculados a las diferentes facultades y dependencias de la universidad: medicina, ciencias naturales, derecho, humanidades (filosofía y filología) y literatura. Esto es complementado con secciones de gran extensión encargadas de reseñar las novedades bibliográficas y hemerográficas, notas sobre la vida universitaria, en las cuales es común que se resaltaran los comentarios en diversos medios sobre la revista y su importancia a la hora de ampliar el catálogo de la biblioteca a través del canje, también es común en estas notas la mención a otras actividades de extensión cultural. Finalmente, hay algunas secciones especializadas a modo de anexos como una sección jurídica y, a partir del número doble 46-47 (1941), unas antologías poéticas que comienza con "Veinte poetas del Brasil contemporáneo" y que luego cambiarán su nombre por "Cuadernillo de poesía".

La Revista de la Universidad Católica Bolivariana tuvo una estructura bastante similar. Ocasionalmente, aunque de manera más frecuente que la revista Universidad de Antioquia, publicó textos de carácter programático, en general, de sus rectores, en los cuales el tema es político-religioso; pero el número típico estuvo conformado por una sección con artículos de fondo que se podrían agrupar principalmente en los campos del derecho, la filosofía, la historia, la política y la sociología (tal como era entendida en esa época en Colombia), aunque también hubo artículos de otros campos cercanos como los estudios

18 "El Premio Vergara y Vergara de literatura nacional", 376. 
del folclor y la geografía. A esta sección en general le seguían reseñas bibliográficas y hemerográficas, las notas que son artículos de menor extensión que los de la primera sección y un apartado de información universitaria sobre las actividades de la institución que incluían tanto su organización interna (compra de terrenos para el campus universitario y diseño de este) como sus actividades de extensión. El primer número de los "Cuadernillos de poesía colombiana” aparecerá en el número doble 8-9 de 1938, es decir, tres años antes que Universidad de Antioquia. En el número 40-41 de 1945, aparecerá por primera vez la sección, también a modo de anexo: "Arte", dedicada a presentar la obra de artistas plásticos colombianos. Es importante señalar que, a pesar de que la jerarquía católica del país y de la ciudad sostenía una confrontación con los gobiernos liberales y sus políticas de extensión cultural, la cual incluía la Revista de las Indias, ${ }^{19}$ en la efímera sección "Revistas de América" del volumen 4, número 14, de 1940, salió una reseña ampliamente positiva de esta publicación. De esta misma valoración será objeto la Revista Universidad de Antioquia en el número 19-20 de 1941.

Las reseñas de revistas y libros evidencian un aspecto, sin duda, importantísimo a la hora de valorar el papel que desempeñaron ambas publicaciones universitarias. La biblioteca de la Universidad de Antioquia contaba a comienzos de 1935 con una reducida colección bibliográfica estimada en 2000 volúmenes, ${ }^{20}$ entre los que se contaban los ejemplares de cuatro revistas extranjeras y tres colombianas; en septiembre del mismo año, se hablaba de "cuatrocientos canjes procedentes de los países más cultos", ${ }^{21}$ y dos años después se señalaba que gracias al canje a la colección se habían sumado cerca de 4000 volúmenes que correspondían a libros, folletos y 965 revistas internacionales y 150 nacionales; la mayoría de estas publicaciones eran de carácter universitario. ${ }^{22} \mathrm{Si}$ bien la cifra puede generar suspicacias y seguramente de muchas revistas solo llegó un número, lo importante es que la difusión cultural no se realizaba solo con los artículos impresos en su página, sino también con la labor de poner a disposición de la comunidad universitaria y la ciudad un gran número de publicaciones que de otro modo serían inaccesibles y que para 1953 se calculaban en 125000 obras, muchas de ellas conseguidas a través del canje de la revista institucional. ${ }^{23}$

Otra forma de extender la cultura por las dos revistas analizadas fue la promoción de otro tipo de actividades de difusión cultural que las universidades realizaban, por ejemplo, fueron constantes las menciones en la Revista de la Universidad Católica Bolivariana de los cursos que esta universidad ofrecía a los obreros y a las damas de la ciudad, además, de información sobre el programa radial La hora católica o de las conferencias que se realizaban en la biblioteca y eran transmitidas por La Voz de Antioquia ${ }^{24}$ o la radiodifusión de La hora bolivariana por la Emisora Claridad:

Por este medio la Universidad llevará a todos los sitios de la nación y de América la voz de sus profesores, de sus estudiantes, de sus directores. En esta hora semanal de radiodifusión se tratarán todos los problemas

19 Renán Silva, "Reforma cultural, Iglesia católica y Estado durante la República Liberal", en República Liberal: Sociedad y cultura, ed. por Rubén Sierra Mejía (Bogotá: Universidad Nacional de Colombia, 2009).

20 "Biblioteca general y revista Universidad de Antioquia", Revista Universidad de Antioquia, no. 112 (1953): 684.

21 "Una visita a la biblioteca de la Universidad de Antioquia", El Colombiano, 13 de septiembre de 1935, p. 9.

22 Clodomiro Ramírez y Alfonso Mora Naranjo, "Algunas anotaciones sobre la Revista Universidad de Antioquia", Revista Universidad de Antioquia, no. 15-16 (1937): 482.

23 "Biblioteca general y Revista Universidad de Antioquia", 685.

24 “Información universitaria", Revista Universidad Católica Bolivariana 4, no. 11-13 (1939): 247. 
más actuales, todos los temas de interés de la patria, todo, en fin, lo que en alguna manera pueda contribuir al engrandecimiento cultural de Colombia y de la Universidad. Así la Universidad estrechará sus vínculos con todo el país y hará más dilatado y accesible el campo de sus servicios. ${ }^{25}$

La cita sirve para resaltar tres aspectos clave de la extensión universitaria: la apuesta por temas que se consideraban de actualidad o de interés general, el afán por cultivar y engrandecer a los colombianos y el deseo de proyectar las universidades por fuera de sus claustros. Infortunadamente, no ha sido posible encontrar fuentes documentales fidedignas sobre la recepción de estas revistas, aunque se ha podido identificar publicidad en otras publicaciones como la Revista Javeriana. ${ }^{26}$ No obstante, esta misma ausencia nos lleva a pensar que su distribución fue restringida, más si se considera que sus artículos fueron en general densos y relativamente extensos, y en esa medida presentaban dificultades de lectura para el lector ocasional o menos formado. En este sentido, ambas revistas eran más semejantes a revistas contemporáneas para intelectuales como la Revista de las Indias, Revista de América, Espiral o Bolívar que a revistas para un público amplio como Cromos, El Gráfico o Estampa, las cuales poseían otro tipo de tipografía, párrafos más cortos, un número mucho mayor de ilustraciones, y se ocupaban de temas menos especializados.

\section{Las emisoras universitarias, 1935-1954}

América Latina, y en especial Argentina, ocupa un lugar singular en los procesos quehan ido constituyendo la radiodifusión en lo que conocemos actualmente. En Buenos Aires, se realizó la primera transmisión radial comercial en 1920 y en abril de 1924 en la Universidad Nacional de La Plata se inauguró Radio Universidad, la cual se convirtió en una emisora pionera en difundir el arte y la educación. Otros centros universitarios como la Universidad Autónoma de México (conocida desde 1945 como Universidad Nacional Autónoma de México) y la Universidad Técnica Federico Santa María, en Chile, ambas en 1937, comenzaron a usar las ondas radiales para amplificar el alcance de las actividades que realizaban en sus claustros.

Se podría decir que, en general, para Colombia, la década de 1930 estuvo signada por un espíritu de formación y consolidación del medio radial, tanto para las llamadas emisoras comerciales como para las que tenían un carácter educativo y cultural como las emisoras universitarias. Para estas últimas, así como en otros países, en este proceso, confluyeron el interés de las universidades por la implementación de estrategias de extensión educativa y cultural, con la curiosidad y el entusiasmo característicos de los años de experimentación con las ondas radiales, pues el personal a cargo de las estaciones transmisoras por lo general eran miembros de sus comunidades estudiantiles. ${ }^{27}$ Por otro lado, no deja de ser llamativo que este tipo de iniciativas se gesten en Popayán y Medellín, pues la primera emisora universitaria de Bogotá comenzó a funcionar solo en 1977.

25 “Información universitaria", Revista Universidad Católica Bolivariana 9, no. 33 (1943): 481.

26 “Revista Universidad Católica Bolivariana”, Revista Javeriana 16, no. 78 (1941): 163.

27 Catalina Castrillón Gallego, Todo viene y todo sale por las ondas: Formación y consolidación de la radiodifusión colombiana, 1929-1954 (Medellín: Universidad de Antioquia, 2015), 102. 
De acuerdo con avisos publicados en 1932 por periódicos como Mundo al Día y El Colombiano, la Universidad del Cauca inauguró en Popayán una emisora que pretendía "prestar servicio a todos los habitantes de la ciudad y no solamente a los universitarios residentes en ella". ${ }^{28}$ La estación contó con la participación de destacados intelectuales de la época como Guillermo Valencia (1873-1943) y César Uribe Piedrahita (1897-1951), al igual que con la colaboración del Ministerio de Industria, que presentaba programas en los que se exaltaba la riqueza de los recursos del país. Desafortunadamente, no se cuenta con información que permita establecer durante cuánto tiempo funcionó.

La Emisora Cultural Universidad de Antioquia tuvo una fase inicial (1933 y 1938) en la que su funcionamiento estuvo a cargo de un pequeño grupo de estudiantes del liceo de la institución liderado por el profesor de física. ${ }^{29} \mathrm{El}$ Ministerio de Correos y Telégrafos le adjudicó licencia de funcionamiento en septiembre de 1937 y al año siguiente recibió la concesión de los equipos de onda larga de la desaparecida emisora Voz Katía que había sido propiedad del Gobierno municipal, con el compromiso de transmitir los actos oficiales de la Alcaldía y las sesiones del Consejo de Medellín. A partir de 1938, comenzó el funcionamiento de la emisora con un horario de transmisión regular, no obstante, todavía carecía de una programación que expresara los propósitos de extensión cultural en tanto voz de la alma mater.

En septiembre de 1948, la Universidad Pontificia Bolivariana, también de Medellín, puso en funcionamiento Radio Bolivariana para ofrecer a la audiencia otra emisora cultural que fuera "trasunto exterior de la cátedra universitaria, para que el pueblo se beneficie de su verbo; la proyección de sus luces aspira a crear la cultura popular, con el sello espiritualista, que es el signo de Dios en nuestra inteligencia, con todo el afán de una obra misionera". Este aspecto quedaba claro en la singular concepción que expresaba de la cultura y la civilización solo comprendidas desde su estrecha relación con las ideas católicas. ${ }^{30}$

Se podría decir que las emisoras eran la voz joven de ambas instituciones, pues, a diferencia de las revistas universitarias que estaban en manos del director de la biblioteca o del rector, desde sus fundaciones hasta 1954 la dirección y el funcionamiento de las estaciones radiales estuvieron a cargo de estudiantes y profesores.

En 1949, Radio Bolivariana y la Emisora Cultural Universidad de Antioquia desarrollaron estrategias editoriales de distribución gratuita, guías o boletines de programación, en los que mes a mes entregaban a sus lectores detalles sobre los diferentes espacios que transmitían y los horarios en que lo hacían. De igual manera, publicaban las novedades relacionadas con aspectos técnicos como la adquisición de equipos, el cambio en las potencias de transmisión o el traslado de los estudios hacia nuevas instalaciones; en ellas, también aparecía el listado del personal técnico que administraba y hacía posible el funcionamiento de las emisoras.

28 "Nueva estación de radio", Mundo Al Día, 3 de junio de 1932, p. 2; "Nueva estación radiodifusora", El Colombiano, 16 de julio de 1932, p. 2.

29 Álvaro Vélez Betancur, "Historia de la Emisora Cultural Universidad de Antioquia, 1938-1948" (tesis de grado, Universidad de Antioquia, 2002), 20.

30 "Radio Bolivariana: Una emisora al servicio de la cultura y civilización católicas que son las únicas que sí merecen este apelativo", Guía de Programas, no. 13 (1950): 23. 
Estas publicaciones resultan significativas porque fueron concebidas como un complemento a las actividades propiamente radiales, lo que les permitió afianzar su relación con los oyentes, puesto que con frecuencia les instaban a enviar sus opiniones y sugerencias sobre lo que estaban escuchando; estas cartas eran bien recibidas y se les daba visibilidad en las páginas de los boletines. Por otro lado, con este tipo de publicaciones, las emisoras expresaban un voto de confianza con sus audiencias, en tanto daban cuenta de la manera en que funcionaban y les permitían dejar claros los principios que orientaban la existencia de las estaciones radiales y sus propósitos como instrumento de extensión cultural para ambas instituciones.

Por los ejemplares que se conservan, es posible decir que la Guía de programas de Radio Bolivariana se publicó durante tres años con cierta irregularidad. En 1949, tuvo una frecuencia mensual y se publicaron nueve ediciones entre marzo y noviembre; el año siguiente, se publicaron cinco entre abril y septiembre. La publicación reaparece en 1958 con dos ediciones, una correspondiente a mayo y junio, y otra a septiembre. Uno de los periódicos de la ciudad reseñó su aparición de la siguiente manera:

Hemos recibido el boletín de la Radio Bolivariana que trabaja en 1.550 kilociclos en onda larga y que viene desarrollando una vasta labor cultual. La gran Universidad Pontificia Bolivariana no ha querido permanecer dedicada únicamente a su labor de enseñanza. Cumple a cabalidad la función de las universidades, y por eso, su revista, su biblioteca, su centro de obreros y ahora su radiodifusora, son contribuciones a la grandeza de Antioquia.

El boletín al que nos referimos, correspondiente a marzo, no se contenta con transcribir los programas diarios, sino que da importantes informaciones sobre el movimiento de la universidad y una explicación sintética de los principales puntos de los programas. ${ }^{31}$

La primera edición del Boletín de programas de la Emisora Cultural Universidad de Antioquia corresponde a mayo de 1949, y a partir de ese momento se publicó sin interrupciones de manera mensual hasta 1954. En su primera nota editorial, expresan que la institución de la que hacía parte comprendía a cabalidad la misión educativa y cultural que debía cumplir y, por ello, había propiciado la creación de recursos que complementaban la labor realizada en las facultades y escuelas de su claustro universitario, tales como la Emisora Cultural, el Instituto Filológico, la imprenta, la biblioteca general, la Revista Universidad de Antioquia y la revista Estudios de Derecho. Se agregaba que durante sus años de funcionamiento la labor de la emisora había sido incomprendida y tenía desafectos, "sin embargo, el medio hostil ha ido cediendo y es así como hoy en día la Emisora Cultural cuenta con un apreciable número de simpatizantes y oyentes, muchos de ellos del núcleo estudiantil, que encuentran en nuestros programas un descanso espiritual en medio de la lucha cotidiana". ${ }^{2}$

Pese a que ambas publicaciones compartían varias características, es importante señalar los rasgos que las diferenciaban, además de la mencionada regularidad con la que aparecieron. Llama la atención la manera en que la Guía de programas de Radio Bolivariana es utilizada para promocionar no solo la emisora, sino también la universidad. Esto es en especial evidente a partir de junio de 1949 cuando comenzaron a

31 "La radio de la UPB está ya transmitiendo", El Colombiano, 9 de marzo de 1949, p. 4.

32 "Sin título", Boletín de programas, no. 1 (1949): 1. 
aparecer en las páginas del boletín anuncios breves en los que se exaltaba la actividad y el impacto de las actividades académicas de la institución, pero al mismo tiempo se buscaba lograr patrocinio económico para la vasta labor social que realizaba la universidad a través de becas para estudiantes de todo el país, así como de la formación gratuita que proporcionaba en los talleres y en el Círculo Obrero a sectores poco favorecidos.

Existen dos asuntos fundamentales para el estudio de las emisoras: el primero tiene que ver con sus horarios de funcionamiento y el segundo con la programación que ofrecían a sus audiencias. Para ello, resulta indispensable la revisión de los boletines de programación porque a partir de ellos es posible reconstruir la parrilla de programación y hacerse una idea de cómo sonaban estas estaciones radiales. Sobre los horarios de funcionamiento, es necesario mencionar que, para el periodo de estudio, el tiempo de transmisión de ambas emisoras se incrementó de forma paulatina, dado que ambas surgieron a partir de la curiosidad y el entusiasmo de estudiantes y profesores.

Como se mencionó, la Emisora Cultural Universidad de Antioquia estaba al aire desde 1933, pero fue a partir de 1938 cuando implementó un horario de transmisión regular. Pasó de funcionar dos horas, entre las ocho y las diez de la noche, a cubrir en la década de 1950 un tiempo de transmisión de ocho horas diarias distribuidas en un segmento diurno de once de la mañana hasta las dos de la tarde, y otro nocturno entre las 6:30 y las 10:30 p. m. Por su parte, durante su primera etapa de funcionamiento, las transmisiones de Radio Bolivariana tenían lugar de lunes a sábado entre las seis de la tarde y las diez de la noche; una década después, en 1958, esta emisora también había implementado dos franjas horarias: una entre las 12:30 y las 2:00 p. m., y otra entre las 6:50 y las 10:15 p. m.

La duración de los programas podía variar entre los quince y los treinta minutos. En casos excepcionales, se extendían a más de una hora. Ambas emisoras desempeñaron un papel importante al abrir sus micrófonos a la transmisión de los actos conmemorativos de diferentes efemérides como los aniversarios de fundación de las respectivas universidades o de las mismas emisoras. Por ejemplo, en octubre de 1950, la Emisora Cultural Universidad de Antioquia anunciaba en su boletín que el 9 transmitiría un programa especial en cadena con la Radio Nacional y las demás estaciones de la ciudad con motivo del día clásico de la universidad y la inauguración de los nuevos equipos e instalaciones de la emisora, además de todos los actos de la semana universitaria y un ciclo de microprogramas con biografías de los rectores. ${ }^{33}$

En cuanto al contenido de los programas, las dos emisoras daban prioridad a los espacios propuestos por sus diferentes dependencias académicas. En la emisora de la Universidad de Antioquia, se transmitía la Revista Jurídica, a cargo de la Facultad de Derecho, además de varios espacios a cargo de la Facultad de Economía; igualmente, en colaboración con la Facultad Nacional de Minas y la Facultad Nacional de Agronomía, los programas Novedades de ingeniería y La universidad en el campo. En Radio Bolivariana, se pueden mencionar Jurisprudencia a cargo de la Facultad de Derecho y Ciencias Políticas, y los programas El laboratorio, IQ y Recortes científicos de la Facultad de Ingeniería Química.

Además, a una amplia oferta de espacios dedicados a la cultura, el arte, la música, la literatura y la lengua, se sumaban los informativos y los ofrecidos por la Alianza Francesa, el Centro Colombo Americano, el Centro Colombo Británico y los cuerpos consulares de países amigos. Es importante llamar la atención

33 "Programa", Boletín de programas, no. 1 (1949): 2. 
sobre la presencia en ambas emisoras de programas como Ventana del deporte y Noticiero deportivo que dan cuenta del deseo de brindar información de interés para el público universitario y general.

Igualmente, es importante resaltar que la emisora de la Universidad de Antioquia dedicara en su programación un espacio semanal para dar a conocer el papel activo que estaban desempeñando las mujeres en la sociedad a partir de su incursión en la educación universitaria y sus conquistas en espacios laborales y profesionales hasta ese momento inéditos: programas como Temas feministas en 1949, Charlas femeninas en 1951 y Mundo femenino en 1954 daban cuenta de ello. En Radio Bolivariana, estos temas también tuvieron cabida en su programación: para 1951, se transmitía el programa Estampas femeninas a cargo de los colegios femeninos de la ciudad.

Otro tema importante para resaltar en la programación durante el periodo de estudio es el relacionado con la divulgación de ideas religiosas. Contrario a lo que podría esperarse por el carácter de la institución a la que pertenece, la Emisora Cultural Universidad de Antioquia tuvo programas semanales como Música religiosa, Música sagrada, Estampas del cristianismo, Hora ignaciana y Orientación espiritual, este último tenía como propósito brindar "formación mística católica a la juventud" ${ }^{34}$ En Radio Bolivariana, este tipo de programas en general tenían mayor presencia y frecuencia. La tabla 1 presenta una síntesis de 1949 a 1950.

Tabla 1.

\begin{tabular}{|c|c|c|c|}
\hline \multicolumn{2}{|c|}{1949} & \multicolumn{2}{|c|}{1950} \\
\hline Noticiero católico & $\begin{array}{l}15 \text { minutos } \\
\text { Lunes a sábado }\end{array}$ & Noticiero católico & $\begin{array}{l}15 \text { minutos } \\
\text { Lunes a sábado }\end{array}$ \\
\hline $\begin{array}{l}\text { Momento juvenil, programa } \\
\text { de la Juventud Católica }\end{array}$ & 15 minutos semanal & Comentaristas católicos & $\begin{array}{l}15 \text { minutos } \\
3 \text { veces a la semana }\end{array}$ \\
\hline La voz de testimonio & 30 minutos semanal & Dramas católicos & $\begin{array}{l}30 \text { minutos } \\
\text { semanal }\end{array}$ \\
\hline & & Biografías de los pontífices & $\begin{array}{l}15 \text { minutos } \\
\text { semanal }\end{array}$ \\
\hline
\end{tabular}

Fuente: elaboración propia.

Sobre Comentaristas católicos mencionaban en la Guía de programas que respondía a la necesidad de emprender "una lucha contra los principios revolucionarios del comunismo que trata de sustituir nuestra organización social por la demagogia. Nadie, sin faltar al sentido común, puede negar el peligro que entraña el sistema marxista. Afortunadamente Colombia, los enemigos del orden social no han podido sacar avante sus propósitos". ${ }^{35}$

Pese al reconocimiento del que gozaban ambas emisoras por la calidad de los programas que transmitían, al igual que por la vastedad y especialización de sus respectivas discotecas, nunca gozaron de una amplia

34 “Nuevos programas", Boletín de programas, no. 55 (1954): 4.

35 “Comentaristas católicos", Guía de Programas, no. 12 (1950): 15. 
audiencia, en parte, por las condiciones técnicas que durante mucho tiempo dificultaron a los oyentes sintonizarlas sin interferencias de otras frecuencias. Incluso, en la década de 1960, la crítica radial continuaba resaltando estas debilidades cuando se ocupaba de las emisoras que podían sintonizarse en la ciudad:

Voz del triunfo; como siempre... del montón... Inventaron el baile de la "Hula-hula"... qué bárbaros.

Reloj: Sintonía, exceso de propaganda y "bachecitos”. Por lo demás bien.

Bolivariana: Con todo y eso no pasa de "emisora experimental".

Nutibara. Lo mismo de siempre.

Siglo XX: Podría ocupar posición privilegiada...

Independencia: Con equipos no basta, hay que mejorar la programación...

UdeA: (Léase Radio Bolivariana). ${ }^{36}$

Esta situación señala una especie de paradoja, pues, a pesar de su marginalidad en el contexto de la radiodifusión local y nacional, las dos emisoras universitarias sobresalían y eran tenidas en cuenta, pese a que contaban con pocas horas de transmisión en comparación con las demás estaciones radiales. Además, fueron esfuerzos relativamente pioneros en nuestro medio.

\section{Conclusiones}

Cultura y más cultura, eso fue lo que ambas universidades a través de sus emisoras, revistas y aulas intentaron entregarle a la ciudad, la región y el país. Al hacerlo, se enfrentaron a sus propias limitaciones, principalmente, a la debilidad de su infraestructura y la escasez de sus recursos para participar en un mercado cada vez más competitivo, profesional, diverso y que se dirigía hacia una dirección decididamente comercial. A esto se sumaban las tensiones y contradicciones inherentes a proyectos de este tipo, que se enfrentaban al reto de difundir expresiones culturales que, paradójicamente, eran consideradas minoritarias y, en ese sentido, difícilmente disfrutables más allá de las personas que poseían una educación formal prolongada.

No obstante, esto no impidió que la Universidad de Antioquia y la Universidad Católica/Pontificia Bolivariana realizaran este esfuerzo y constituyeran desde temprano un ecosistema de extensión cultural complejo, en el cual se coordinaban diferentes medios que apelaban tanto a las actividades presenciales y en vivo como a las conferencias, sin dejar de lado actividades mediadas impresas o sonoras, e, incluso, formas mixtas como las conferencias radiofónicas. A estas actividades se sumaron instituciones como las bibliotecas y otras prácticas como los cursos de extensión. Todo esto hace posible afirmar que, a pesar de que el alcance de las emisoras y las revistas fue limitado, ambos medios fueron indispensables para impulsar y sostener este ecosistema, que, aunque no fue mayoritario, ha logrado sobrevivir hasta nuestros días alcanzando en sus más de ochenta años de existencia a un gran número de personas y participando en la configuración de las prácticas culturales locales y regionales.

36 Opel, “Ondas radiales", Pantalla, diciembre de 1963, p. 14. 


\section{Bibliografía}

Arias Trujillo, Ricardo. El episcopado colombiano: Intransigencia y laicidad (1850-2000). Bogotá: Universidad de los Andes, 2003.

"Biblioteca General y Revista Universidad de Antioquia". Revista Universidad de Antioquia, no. 112 (1953): 683-685.

Castrillón Gallego, Catalina. Todo viene y todo sale por las ondas: Formación y consolidación de la radiodifusión colombiana, 1929-1954. Medellín: Universidad de Antioquia, 2015.

"Comentaristas católicos". Guía de Programas, no. 12 (1950): 15.

Deas, Malcolm. "Las claves del periodo". En Colombia: Mirando hacia dentro. Vol. 4: 1930-1960, coordiando por Eduardo Posada Carbó y Deas Malcolm, 17-24. Bogotá: Taurus, 2015.

“Definición”. Revista Universidad Católica Bolivariana 1, no. 1 (1937): 3.

"El Premio Vergara y Vergara de literatura nacional". Revista Universidad Católica Bolivariana 1, no. 3 (1937): $376-$ 377.

"En homenaje a monseñor Sierra". Revista Universidad de Antioquia, no. 44 (1941): 543-544.

"Información universitaria". Revista Universidad Católica Bolivariana 4, no. 11-13 (1939): 239-251.

"Información universitaria". Revista Universidad Católica Bolivariana 9, no. 33 (1943): 480-484.

"La radio de la UPB está ya transmitiendo". El Colombiano, 9 de marzo de 1949, p. 4.

"La universidad y la cultura". Revista Universidad de Antioquia, no. 103 (1951): 589-590.

Londoño Vélez, Santiago. "La Revista Universidad de Antioquia". Boletín Cultural y Bibliográfico 26, no. 18 (1989): 42-49.

Montaldo, Graciela. Museo del consumo: Archivos de la cultura de masas en Argentina. Buenos Aires: Fondo de Cultura Económica, 2016.

"Nueva estación de radio". Mundo Al Día, 3 de junio de 1932, p. 2.

"Nueva estación radiodifusora". El Colombiano, 16 de julio de 1932, p. 1.

"Nuevos programas". Boletín de programas, no. 55 (1954): 4.

Opel. "Ondas radiales". Pantalla, 20 de diciembre de 1963, p. 14.

"Programa”. Boletín de programas, no. 17 (1950): 2.

Rama, Ángel. La ciudad letrada. Santiago Chile: Tajamar, 2004.

"Radio Bolivariana: Una emisora al servicio de la cultura y civilización católicas que son las únicas que sí merecen este apelativo". Guía de Programas, no. 13 (1950): 23.

Ramírez, Clodomiro. "Nuestros propósitos". Revista Universidad de Antioquia, no. 1 (1935): 5-6.

Ramírez, Clodomiro y Alfonso Mora Naranjo. "Algunas anotaciones sobre la Revista Universidad de Antioquia". Revista Universidad de Antioquia, no. 15-16 (1937): 479-86.

República de Colombia. Memoria de Educación Nacional 1939. Bogotá: Imprenta del Estado Mayor General, 1939.

"Revista Universidad Católica Bolivariana". Revista Javeriana 16, no. 78 (1941): 163.

Romero, José Luis. Latinoamérica: Las ciudades y las ideas. Medellín: Universidad de Antioquia, 1999.

Sierra, José J. “Radiodifusión cultural”. Revista Universidad de Antioquia, no. 13 (1937): 31-39.

Silva, Renán. "La cultura". En Colombia: Mirando hacia dentro. Vol. 4 1930-1960, coordinado por Eduardo Posada Carbó y Malcolm Deas, 265-329. Bogotá: Taurus, 2015.

Silva, Renán. "Reforma cultural, Iglesia católica y Estado durante la República Liberal". En República Liberal: Sociedad y cultura, editado por Rubén Sierra Mejía, 223-266. Bogotá: Universidad Nacional de Colombia, 2009.

Silva, Renán. República Liberal, intelectuales y cultura popular. Medellín: La Carreta, 2004.

"Sin título". Boletín de programas, no. 1 (1949): 1.

"Una visita a la biblioteca de la Universidad de Antioquia". El Colombiano, 13 de septiembre de 1935, p. 9.

Vélez Betancur, Álvaro. "Historia de la Emisora Cultural Universidad de Antioquia, 1938-1948”. Tesis de grado, Universidad de Antioquia, 2002. 